\title{
Phase Tracking Sequences for 5G NR in 52.6-71 GHz Band: Design and Analysis
}

\author{
Alexander MALTSEV ${ }^{\mathrm{a}, 1}$, Andrey PUDEEV ${ }^{\mathrm{b}}$, Seonwook KIMc, Suckchel YANG ${ }^{\mathrm{c}}$, \\ Seunghwan $\mathrm{CHOI}^{\mathrm{c}}$, Sechang $\mathrm{MYUNG}^{\mathrm{c}}$ \\ ${ }^{a}$ Nizhny Novgorod State University, Nizhny Novgorod, Russia \\ ${ }^{\mathrm{b}}$ LG Electronics Russia R\&D Lab, Moscow, Russia \\ ${ }^{\mathrm{c}}$ LG Electronics, Seoul, Korea
}

\begin{abstract}
This paper presents a novel approach to the phase tracking reference signal (PTRS) design for phase noise impact compensation in the $5 \mathrm{G}$ NR communication systems intended to work in a new $52.6 \mathrm{GHz}$ to $71 \mathrm{GHz}$ frequency band. For detailed problem illustration, the phase noise compensation algorithms are discussed and explained, from the basic common phase error (CPE) compensation to the MMSE-base inter-carrier interference (ICI) filtering. Performance of the different phase noise compensation algorithms is investigated for the baseline PTRS accepted in the current 5G NR specification and compared with the newly proposed approach to the PTRS design. This approach is based on nulling the subcarriers adjacent to the reference signals to minimize influence of the ICI on the estimation process. It was shown that new nulling PTRS design outperforms currently used distributed PTRS structure. In addition, numerical results represent a trade-off between the filter size and the amount of the allocated training resources to achieve better performance. It was shown that proposed PTRS structures and processing algorithms give ICI compensation level very close to optimal scheme and thus, different approaches (such as time domain compensation) may be required for further progress.
\end{abstract}

Keywords. 5G NR, beyond $52.6 \mathrm{GHz}$, CPE, de-ICI filtering, OFDM, PTRS design, phase tracking, phase noise

\section{Introduction}

Recent advancements in the communication technologies and an ever-growing data traffic capacity demands drive the $5 \mathrm{G}$ NR towards higher frequency bands and subjected a new work item on the support of the frequencies from $52.6 \mathrm{GHz}$ to $71 \mathrm{GHz}$ [1]. It is noted [2] that one of the key challenges for the OFDM systems in the millimeter-wave bands is a phase noise that causes severe inter-carrier interference (ICI) and prohibits the operation of the spectrally efficient higher order modulations without applying a specific phase tracking and ICI compensation algorithms. The performance of these algorithms strongly depends on training sequence design and functions. Although such phase tracking sequences are already specified in the $5 \mathrm{G} \mathrm{NR}$ standards, the expansion to the $52.6-71 \mathrm{GHz}$ band may require reconsideration of the currently implemented sequence structures.

\footnotetext{
${ }^{1}$ Corresponding author: Alexander Maltsev, e-mail: maltsev@rf.unn.ru.
} 
In 5G NR specifications [3], phase tracking reference signal (PTRS) is introduced for mitigating phase noise impact and is defined as the known pseudo-random QPSKmodulated training signal. PTRS can be allocated within a time-frequency resource grid with configurable parameters for periodicity (or density) in time and frequency. In particular, the frequency domain parameter $\mathrm{K}$ and the time domain parameter $\mathrm{L}$ correspond to PTRS mapping for which PTRS resource element is allocated for every $\mathrm{K}$-th resource block in frequency domain and for every L-th OFDM symbol in time domain. Variation of these parameters may affect the accuracy of the phase estimation and phase noise compensation.

The impact of phase noise on the OFDM system performance is well studied in the literature using theoretical and simulation analysis based on a different noise models [4]. For OFDM systems, phase noise causes so-called Common Phase Error (CPE) shift for all subcarriers in the signal band and, secondly, to the loss of subcarrier orthogonality due to the ICI effect. For reliable system operation, especially using the higher order modulations, these effects should be compensated. It should be noted that for lower order modulation, typical values of additive (thermal) noise are significantly larger than phase noise influence, and the latter can be neglected.

The CPE term may be efficiently estimated with the uniform PTRS grid specified in the Rel-15 [3] using relatively simple processing. This may be especially efficient in the multipath environment with the frequency-selective channel.

On the contrary, the ICI term is more difficult to estimate and compensate than the CPE term. Such processing typically requires per-symbol matrix inversion operation to obtain LS or MMSE estimation of the phase noise realization and further (de)convolution of the received signal to obtain noise-free data in the frequency domain - for example as in [5][6].

A perspective direction may be a design of the specialized PTRS structures, which is more suitable for the ICI compensation in the sense of performance and implementation complexity. In the recent works [7][8] a block PTRS structure is proposed and analyzed. With such approach, the PTRS resource elements are grouped in chunks, so the neighboring REs interference can be known. A new approach, based on similar principle but different implementation is proposed in present paper. Considered PTRS structure design is based on the adjacent subcarrier nulling and allows not only reducing the number of operations but also isolating PTRS from the random data influence.

An important role in the phase noise impact on the OFDM signals plays the ratio between the phase noise bandwidth and the OFDM subcarrier spacing (SCS) which in 5 G NR can be largely varied [9]. The part of the phase noise inside the SCS affects only the given subcarrier and causes the phase shift error (or CPE). The part of the phase noise outside the SCS affects neighboring subcarriers and causes ICI.

Figure 1 shows the power spectral density (PSD) of the phase noise, according to the model currently accepted as a baseline for simulations and analysis [10]. For convenience, 5G NR SCS values (including those that are not supported in current NR specification but discussed to be introduced for frequency range from $52.6 \mathrm{GHz}$ to 71 $\mathrm{GHz}$ ) are shown along the frequency axis.

It can be seen that the amount of out-of-SCS noise corresponding to the ICI term is different for different subcarrier spacing, so the higher SCSs are much less susceptible 
to the ICI effect of the phase noise. These qualitative observations further will be verified by qualitative analysis using the link layer simulations of the $5 \mathrm{G}$ NR system.

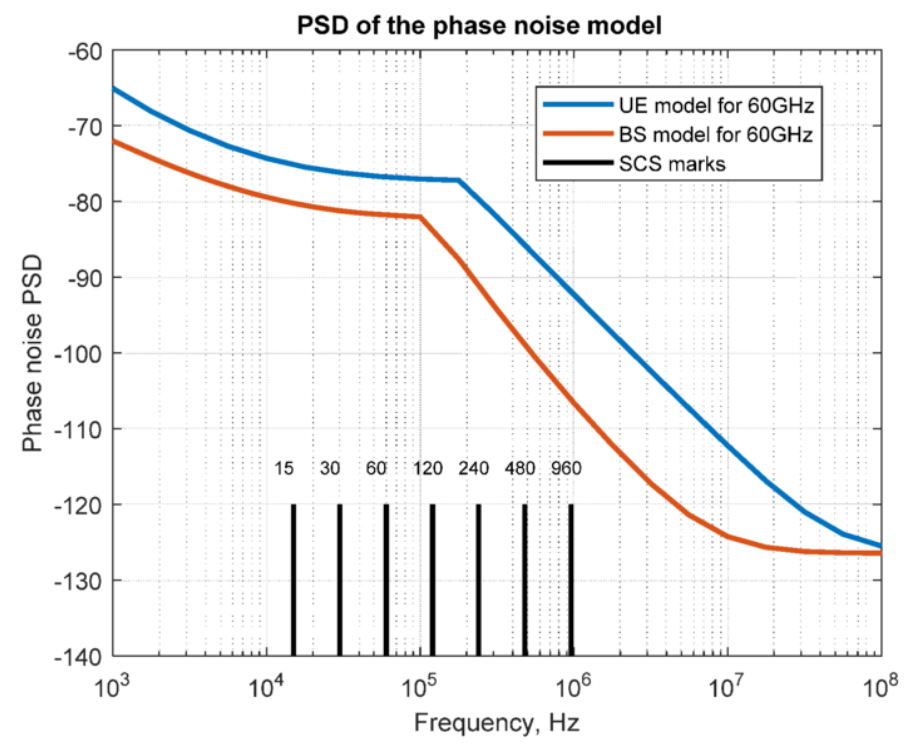

Figure 1 PSD of the phase noise based on model adopted in 3GPP document [10]

\section{Phase noise impact on the OFDM signals}

Analysis of the phase noise influence on an OFDM system performed in a number of works [4][5][6] show that the overall phase noise impact can be divided in the two components - the CPE, and the ICI - see Eq. (1), in details derived in [11]. The first term $\bar{J}_{0}$ is common for all subcarriers on the given OFDM symbol, but largely fluctuating for different symbols. The second term describes influence of the adjacent OFDM subcarriers on the given one due to signal spreading caused by multiplicative phase noise.

$$
Y_{k}=H_{k} S_{k} \bar{J}_{0}+\sum_{i=0, i \neq k}^{N-1} H_{i} S_{i} \bar{J}_{k-i}+\eta_{k}^{A W G N},
$$

where $S_{k}$ is the symbol transmitted at the subcarrier with index $k$, through channel with $H k$ transfer function value at the same subcarrier and affected by additive noise $\eta_{k}^{A W G N}$. Convolution is performed over all $N$ subcarriers, and term $\bar{J}_{k}$ is the phase noise realization in frequency domain at the $k$-th subcarrier defined as:

$$
\bar{J}_{k}=\frac{1}{N} \sum_{n=0}^{N-1} e^{j \phi_{n}} e^{-j 2 \pi n k / N}
$$

In the general communication system phase noise occurs at the transmit side for the carrier frequency upconversion, and at the receive side for the downconversion, so strictly, there are two different phase noises - transmit and receive. For the flat AWGN channel, obviously, they are combined in one, with the same distribution and increased 
power. For the frequency selective channel, the transmit noise is affected by the channel transfer function and thus, may not be described by simple convolution. Fortunately, as shown in [11], the representation of the phase noise impact as convolution in the frequency domain for the case of frequency-selective channels is applicable inside the channel coherence bandwidth, which is typically much larger than phase noise bandwidth, and thus representation (1) can be freely applied for the description of the phase noise impact on the $5 \mathrm{G}$ NR communication system in the considered channels with the delay spread up to tens of nanoseconds. Moreover, the PN spectrum realization has specific symmetry, where the $J_{i}$ and $J_{-i}$ tones taken at the symmetric positions around the DC component $J_{0}$ have equal imaginary parts and real parts with equal magnitudes, but inverted signs [11]. This important property will be used for improvement of the phase noise realization estimate.

Comparing the phase noise bandwidth with the OFDM signal subcarriers spacing (see Figure 1), or, in time domain, comparing the channel coherence time and OFDM symbols duration, we can observe that phase noise is not correlated on the adjacent symbols and thus, phase noise should be estimated and compensated on every OFDM symbol. The 5G NR specification allows changing also time density of the PTRS elements, but for considered SCSs/OFDM symbols durations, PTRSs should be allocated on every OFDM symbol for proper phase noise effects compensation. However, for the purposes of the carrier phase drifts tracking in the cases when phase noise is negligible, the PTRSs allocation can be much sparser.

While the first term in (1) may be rather easily mitigated by finding a common phase shift for the OFDM symbol, the compensation of the ICI may require more advanced algorithms and pilot structures. Current 3GPP 5G NR specifications define the PTRSs as a grid of selected resource elements (REs), sparsely distributed in the frequency domain in every $2^{\text {nd }}$ or $4^{\text {th }}$ resource block of 12 subcarriers (i.e., one PTRS element every 24-th or 48-th subcarrier). Whether it is enough or not, or should we consider another PTRS structures besides distributed grid - this is the subject of present paper studies.

\section{Per-symbol Phase noise compensation approaches}

\subsection{Common phase error (CPE) approximation}

The common phase error term $\bar{J}_{0}$ in (1) is equivalent to the same phase shift of the time-domain representation of the signal, constant during the OFDM symbol. So, the approximation of the phase noise with $\mathrm{CPE}$ in frequency domain is equivalent to the piece-wise constant per symbol phase noise approximation in the time domain [12]. Obviously, this approximation becomes more accurate for smaller symbol durations (i.e., for larger SCSs).

\subsection{Linear trend approximation}

The next logical step in the accuracy improvement of the phase noise evaluation and compensation is using a linear time domain approximation of the phase noise. In practice, such schemes can be implemented by exploitation on the cyclic prefix (CP) 
properties on the OFDM signal, but in this preliminary analysis we assume ideal cases both for piecewise-constant and piecewise-linear approximations.

Figure 2 illustrates the principle and relative accuracy of the constant and linear approximations for different SCSs / OFDM symbol duration values. It can be seen that for higher subcarrier spacing value / shorter OFDM symbols, the constant approximation becomes more and more accurate.

For better understanding of the effects, we will plot the original phase noise PSD estimation versus the PSDs for the noise with compensated constant and linear trends for different SCS sets. The PSD is estimated over large number of realizations using Welch periodogram method [13]. The estimated PSDs in Figure 3 illustrate that estimation and compensation of the CPE works like filtering low frequency part of the phase noise, from the zero frequency to the SCS value. It can be seen that using the 960 $\mathrm{kHz}$ SCS allows more efficient 'filtering' with the larger bandwidth. The linear trend compensation allows even better phase noise compensation, but all these methods do not compensate "out-of-band" noise which implies the noise in the frequencies larger than the SCS and causes ICI, as it can be seen in Figure 3.

So, phase noise compensation algorithms that operate on a per-symbol basis have limited bandwidth and thus, cannot fully compensate the noise impact. However, with the increasing of the SCS value, simple CPE compensation becomes more and more efficient since phase noise quickly degrades with frequency (approximately $30 \mathrm{~dB}$ per decade).

For the baseline subcarrier spacing values, as well as for newly proposed, additional intra-symbol processing is required for the compensation of the higher frequencies phase noise components that will cause ICI.

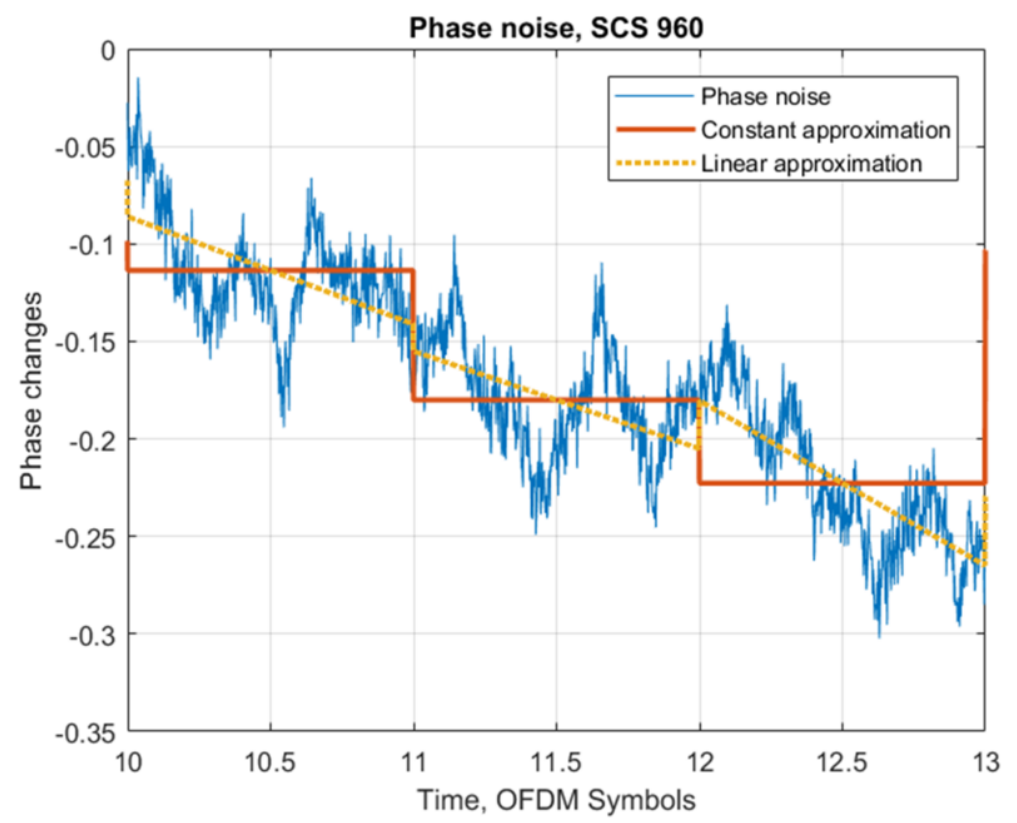

Figure 2 Piecewise constant linear approximations of the phase noise for SCS $960 \mathrm{kHz}$ 


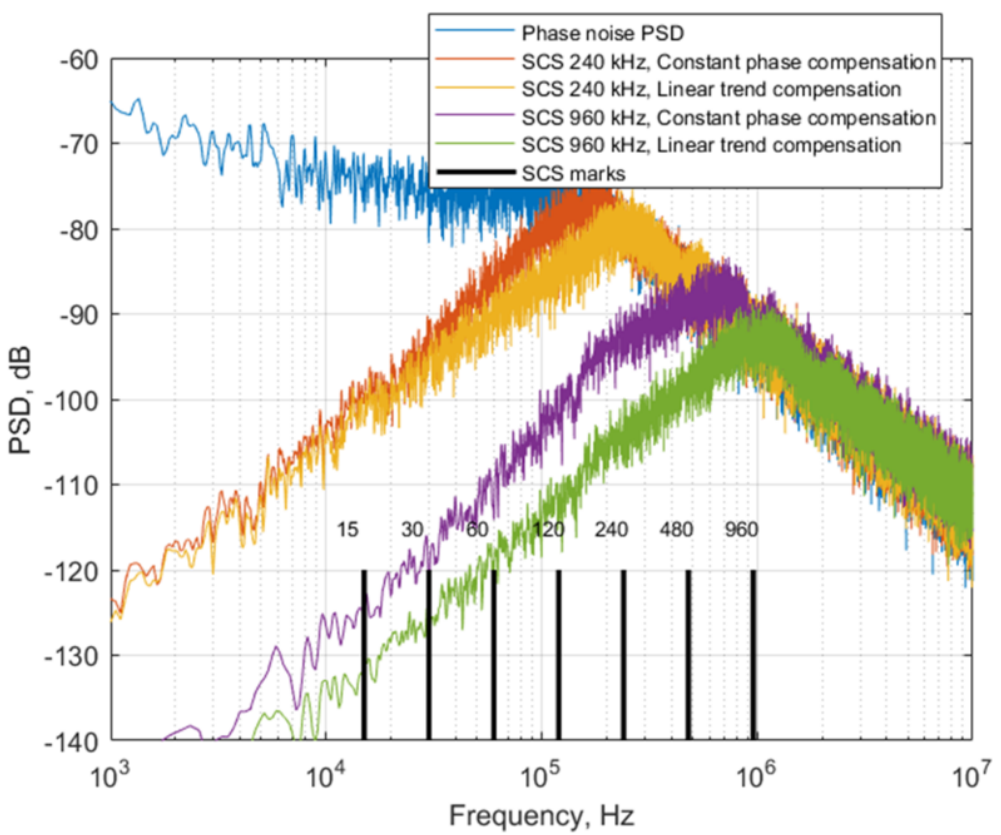

Figure 3 Power spectral density comparison of different trend compensation techniques

\section{Inter-carrier interference compensation schemes}

For the full compensation of the phase noise impact, the exact knowledge of the frequency domain phase noise realization at the OFDM symbol, given by Eq.(2) is required. However, as it can be seen from PSD, frequency domain representation $\bar{J}_{i}$ has the most power in the center and several neighboring subcarriers, whilst the noise influence outside those several subcarriers are much smaller and well behind the additive phase noise. Thus, it is convenient to represent the Eq. (1) as a convolution of the received signal with the 'significant' part of the phase noise, occupying $u$ positions on the both sides of the considered $k$-th subcarrier:

$$
Y_{k}=\sum_{i=-u}^{u} \bar{J}_{i} H_{k-i} S_{k-i}+\eta_{k}^{A W G N}+\eta_{k}^{I C I}
$$

or, substituting the product of transmitted signal and channel at $k$-th subcarriers as $X_{k}$ and equivalent AWGN and residual ICI noise as $W_{k}$ :

$$
Y_{k}=\sum_{i=-u}^{u} \bar{J}_{i} X_{k-i}+W_{k}
$$

In this representation, the significant part of the phase noise is represented as $2 u+1$ tap filter. Since phase noise realization in frequency domain has unity amplitude, the convolution in Eq. (4) is reversible and can be done by filtering with the complex conjugate vector $\bar{J}_{i}$. 
However, the direct solution of the Eq. (4) regarding $\bar{J}_{i}$ requires the contiguous pilot structures [12], whilst in the 5G NR specifications the uniformly sparse grid is considered. To avoid the limitations of the signal structure, instead of estimation of the phase noise realization $\bar{J}_{i}$ using Eq.(4), with the necessity to know adjacent values of $X_{k}$ we can look for the filter that directly transforms the received signal $Y_{k}$, corrupted by the phase noise into the known ideal reference signal values. In this case problem can be formulated as a convolution of the received signal, which is known both at the pilot and data locations with the estimated filter:

$$
X_{k}=\sum_{i=-u}^{u} J_{i} Y_{k-i}+Q_{k}
$$

Solving the Eq. (5) should provide filter with $2 u+1$ taps that can restore the original signal $X_{k}$ from the received signal $Y_{k}$ corrupted by the phase noise. Obviously, increasing the number of filter taps may increase compensation efficiency, since less and less noise appears in the uncompensated part $Q_{k}$. However, with limited observation points, increasing the number of estimated parameters may quickly decrease estimation accuracy, while gains from adding more taps are very small.

Convolution (5) can be rewritten in the matrix form using special matrix structure for the set of $L$ observed subcarriers:

$$
\left[\begin{array}{c}
X_{k_{0}} \\
\vdots \\
X_{k_{L-1}}
\end{array}\right]=\left[\begin{array}{ccc}
Y_{k_{0}+u} & \ldots & Y_{k_{0}-u} \\
\ldots & \ldots & \ldots \\
Y_{k_{L-1}+u} & \ldots & Y_{k_{L-1}-u}
\end{array}\right]\left[\begin{array}{c}
J_{-u} \\
\vdots \\
J_{u}
\end{array}\right]+\left[\begin{array}{c}
Q_{k_{0}} \\
\vdots \\
Q_{k_{L-1}}
\end{array}\right]
$$

or:

$$
\mathbf{X}=\mathbf{Y J}_{u}+\mathbf{W}
$$

Problem (7) has well-known in the literature (for example, [14]) solutions that can be selected on the base of prior knowledge.

The simplest solution, least squares (LS) minimized the squared error of filter tap estimate without any knowledge of the phase noise and AWGN parameters:

$$
\widehat{J}_{u}^{L S}=\left(\mathbf{Y}^{H} \boldsymbol{Y}\right)^{-1} \mathbf{Y}^{H} \mathbf{X}
$$

Minimum mean square error (MMSE) solution:

$$
\widehat{J}_{u}^{M M S E}=\mathbf{R}_{J J} \mathbf{Y}^{H}\left(\mathbf{Y R}_{J J} \mathbf{Y}^{H}+\mathbf{R}_{W W}\right)^{-1} \mathbf{X}
$$

where ()$^{H}$ denote Hermitian transpose, $\mathbf{R}_{\boldsymbol{J} \boldsymbol{J}}$ is the frequency domain phase noise correlation matrix, and $\mathbf{R}_{\boldsymbol{W} W}$ is equivalent noise (residual ICI and AWGN) correlation matrix. Calculation of these values is described in the Appendix A.

MMSE requires prior knowledge of the correlations and noise statistics, has larger complexity in terms of matrix inversions operations, but provides more reliable solution, equally suitable for low and high SNR cases.

Both the LS and MMSE estimates can be further improved by exploitation of the symmetry property of the phase noise frequency domain realization and also de-ICI filter. For this, taps of the estimated filter should be averaged taking into account their symmetry around the center tap. 


\section{PTRS with subcarrier nulling allocation}

It should be noted that both aforementioned algorithms of the phase noise compensation using PTRS are agnostic to the resource allocations - i.e. pilots can be allocated with any pattern along the signal bandwidth. The most efficient way is allocating pilot resources uniformly, to cope with channel frequency selectivity and ensure reliable phase tracking.

However, in this case PTRS values are affected by the ICI from adjacent data subcarriers, induced by the phase noise and other sources. Due to randomness of the data, the full cancellation of the ICI is possible only for decision-directed schemes, with successive decoding and feedback. The computational complexity of such schemes is prohibitive, especially for the millimeter-wave transmissions which typically have very large bandwidth.

Instead, it is possible to allocate PTRSs in a small groups of 3, 5 or generally $(2 n+1)$ subcarriers, with only the center subcarrier active and modulated, and the edge subcarriers nulled. The power from the nulled subcarriers can be effectively reallocated to the center one, increasing the SNR and avoiding the ICI at the same time.

Figure 4 shows the basic principle of distributed PTRS allocation, currently used in 5 G NR specification (in Figure 4(a)) and the newly proposed PTRS with nulling structure (in Figure 4(b)). It should be noted that typically the distance between PTRSs is no less than 24 subcarriers, but for illustrative purposes, the distance is decreased.

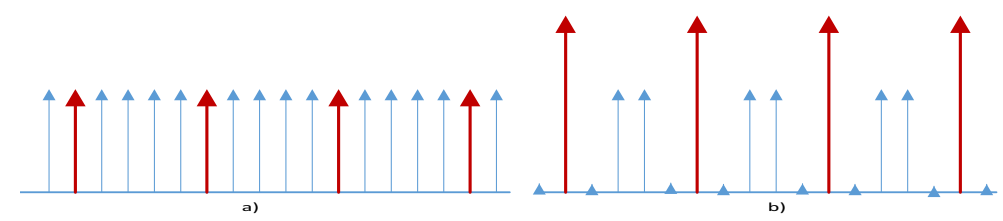

Figure 4 Conventional distributed PTRS allocation (a) vs. PTRS allocation with nulling (b)

It should be noted, that despite nulling of some PTRS subcarriers, they are still counted as reference signals and should be taken into account in the calculations. On the one hand, those subcarriers are included in the calculation of the $\left(\mathbf{Y}^{H} \boldsymbol{Y}\right)$ matrix that is subject to the inversion. Nulled elements on off-diagonal positions make this matrix better conditioned, which is very important in the case of small data allocations, when number of available PTRSs is small. On the other hand, nulled subcarriers significantly decrease the number of multiplications on the final step of Eq. (8) - multiplication by the reference signal $\mathbf{X}_{\mathbf{k}}$.

\section{Computation complexity analysis}

Computation complexity comparison of the considered algorithms may be done by calculating the number of complex multiplications (cmult) required for each algorithm, assuming that $\mathrm{MxN}$ by $\mathrm{NxM}$ matrix multiplication requires $\mathrm{M}^{\wedge} 2 \mathrm{~N}$ scalar operations. It is also assumed that matrix inversion requires the number of multiplications roughly equal to $\mathrm{N}^{\wedge} 3$. Computational complexity for each approach is calculated assuming that 
matrix operations are performed in optimal order, in accordance with corresponding Eq. (8) and (9). Additionally, the number of operations required for application of the filter to the received data is calculated as product of number of data subcarriers by filter size and the shown in a separate column, but also included in the total.

Table 1 shows the number of the complex multiplication required to apply corresponding algorithm, last two columns contain percentage comparison with respect to the base LS scheme. It can be seen that for the large allocations sizes (larger RB number) MMSE becomes very computationally burdened, while for the smaller allocations and larger filter sizes it can be comparable to the LS. The nulling scheme demonstrates a small improvement, since the main part of the complex operations for the LS case involves the application of the filter to the data sequence, and estimation of the filter taps requires much less computations.

Table 1 Computational complexity summary

\begin{tabular}{|c|c|c|c|c|c|c|c|c|c|}
\hline \hline \# RB & $\begin{array}{c}\text { PTRS } \\
\text { K }\end{array}$ & $\begin{array}{c}\# \\
\text { Taps }\end{array}$ & $\begin{array}{c}\# \\
\text { RE }\end{array}$ & $\begin{array}{c}\text { De-ICI } \\
\text { filter, } \\
\text { CMULT }\end{array}$ & $\begin{array}{c}\text { LS, } \\
\text { total, } \\
\text { CMULT }\end{array}$ & $\begin{array}{c}\text { LS+Null, } \\
\text { total, } \\
\text { CMULT }\end{array}$ & $\begin{array}{c}\text { MMSE, } \\
\text { total, } \\
\text { CMULT }\end{array}$ & $\begin{array}{c}\text { LS+Null, } \\
\%\end{array}$ & $\begin{array}{c}\text { MMSE } \\
\%\end{array}$ \\
\hline \hline 8 & 2 & 3 & 96 & 288 & 376 & 365 & 504 & $-3 \%$ & $34 \%$ \\
\hline 8 & 4 & 3 & 96 & 288 & 350 & 345 & 374 & $-1 \%$ & $7 \%$ \\
\hline 8 & 2 & 7 & 96 & 672 & 1292 & 1271 & 1484 & $-2 \%$ & $15 \%$ \\
\hline 8 & 4 & 7 & 96 & 672 & 1178 & 1167 & 1218 & $-1 \%$ & $3 \%$ \\
\hline 64 & 2 & 3 & 768 & 2304 & 2756 & 2671 & 39620 & $-3 \%$ & $1338 \%$ \\
\hline 64 & 4 & 3 & 768 & 2304 & 2548 & 2505 & 7668 & $-2 \%$ & $201 \%$ \\
\hline 64 & 2 & 7 & 768 & 5376 & 7592 & 7421 & 48552 & $-2 \%$ & $540 \%$ \\
\hline 64 & 4 & 7 & 768 & 5376 & 6680 & 6595 & 12824 & $-1 \%$ & $92 \%$ \\
\hline
\end{tabular}

\section{Simulation assumptions and results}

The performances of the considered PTRS allocations were investigated for the SCS of 120, 480 and $960 \mathrm{kHz}$ in the $400 \mathrm{MHz}$ bandwidth. Two PTRS configurations were considered: the 'dense' case with PTRS allocated every $2^{\text {nd }}$ resource block $(K=2)$ and 'sparse' case with PTRS allocated every $4^{\text {th }}$ resource block $(K=4)$. For example, for SCS $480 \mathrm{kHz}$, for the dense case 32 PTRS resource elements were allocated out of 384 total REs for every OFDM symbol, both for baseline and nulling allocations.

To keep the comparison between two different PTRS allocations fair, the amount of resource elements allocated for PTRS (including nulled REs) was the same for every compared scheme, so the amount of active and nulled subcarriers for the case of nulling was equal to the number of PTRS REs in the baseline 5G NR case. Thus, the number of active PTRS REs is decreased for the case of nulling scheme. The power is also equalized, by boosting the center active pilots. 
An OFDM signal with a TX-side phase noise was propagated through a frequency selective channel described by TDL-A model [15], with normalized delay spread of 10ns and Jakes Doppler spectrum corresponding to $3 \mathrm{~km} / \mathrm{h}$. RX-side phase noise is applied after the channel and the thermal AWGN noise is added further. With two receive antennas, 2x2 MIMO system but with a rank-1 transmission was modeled, with the optimal transmit beamforming and receiver MRC processing. Transmit beamforming was performed per precoding block of 24 contiguous subcarriers, assuming SVD decomposition of known channel. At the receiver, per precoding block channel estimation is performed, with the LS fitting to the adaptively selected number of channel impulse response taps.

To access block error rate (BLER) metric, the transmitted signal is encoded / decoded with the LDPC algorithm in accordance with the 5G NR specification [16]. Modulation-coding scheme 28, corresponding to the 64-QAM modulation with coding rate 0.9258 (see [3]) is used. For convenience, simulation parameters and assumptions were summarized in Table 2.

Table 2 Simulation parameters and assumptions

\begin{tabular}{|c|c|c|}
\hline \multicolumn{2}{|c|}{ Parameters } & Assumption \\
\hline \multicolumn{2}{|c|}{ Carrier frequency } & $60 \mathrm{GHz}$ \\
\hline \multicolumn{2}{|c|}{ Bandwidth } & $400 \mathrm{MHz}$ \\
\hline \multicolumn{2}{|l|}{ SCS } & $480 \mathrm{kHz} / 960 \mathrm{kHz}$ \\
\hline \multicolumn{2}{|c|}{ Data allocation (PDSCH) } & 64 / 32 RBs per slot \\
\hline \multirow{2}{*}{$\begin{array}{l}\text { PTRS configuration: } \\
\text { Number of REs }\end{array}$} & $\mathrm{K}=2, \mathrm{~L}=1$ & 32 / 16 REs per symbol \\
\hline & $\mathrm{K}=4, \mathrm{~L}=1$ & 16 / 8 REs per symbol \\
\hline \multicolumn{2}{|c|}{ Channel model/Pathloss } & TDL-A, $10 \mathrm{~ns}$ DS, $3 \mathrm{~km} / \mathrm{h}$ \\
\hline \multicolumn{2}{|c|}{ Transmission scheme } & $2 \times 2$ MIMO \\
\hline \multicolumn{2}{|c|}{ Channel estimation } & LS DFT fitting \\
\hline \multicolumn{2}{|c|}{ Modulation and coding } & 64-QAM, LDPC R=948/1024 \\
\hline
\end{tabular}

Besides the considered practical CPE and LS / MMSE ICI compensation algorithms, two additional reference cases are also evaluated. The first reference case is the straight forward ideal situation without phase noise - the maximum achievable performance in the case of full phase noise cancellation. The second reference case shows the maximum achievable noise cancellation for a given ICI cancellation filter size. It can be called Clairvoyant algorithm for n-taps filter size. Degradation of this reference curve in comparison with ideal no noise case shows the amount of phase noise not covered by de-ICI filter. This gap can be improved only by increasing the filter size.

On the contrary, difference between Clairvoyant and practical LS / MMSE algorithms for a given filter size is caused only by the taps estimation errors and can be reduced by increasing the estimation accuracy - with application of advanced algorithms and increasing the PTRS density.

MCS 28 is selected as an edge performance mode with high spectral efficiency that requires relatively high SNR values to function. With this MCS, ICI compensation in addition to simple CPE processing is required to achieve target 1\% BLER. With lower MCS values, $\mathrm{CPE}$ and phase noise compensation schemes may have only a quantitative 
difference with a couple of $\mathrm{dB}$ gap, while for selected MCS the difference may be qualitative.

Figure 5 and Figure 6 shows the BLER performance under described assumptions for the different SCS values.

The left graph shows BLER curves for the case of $480 \mathrm{kHz}$ SCS, while the right corresponds to $960 \mathrm{kHz}$ SCS, both for baseline value of PTRS density parameter equal to 4 , unless curve legend states another. There are five different algorithms/cases depicted: the black line stands for the ideal case of the phase-noise free system, while the next cyan set of curves correspond to the next degree of idealization: the Clairvoyant algorithm with ideal estimation of the fixed size ICI compensation filter.

The blue and magenta curves show the ICI compensation with filters estimated with the LS and MMSE algorithms, respectively, while red lines with the worst performance illustrate the simplest CPE compensation approach.

Inside this categorization, the curves with different additional parameters like number of filter taps or density parameter values can be distinguished by markers and dashed/dotted line style.

It can be seen that with nulled PTRS allocation, simple LS algorithm reaches the same performance as more complex MMSE algorithm.

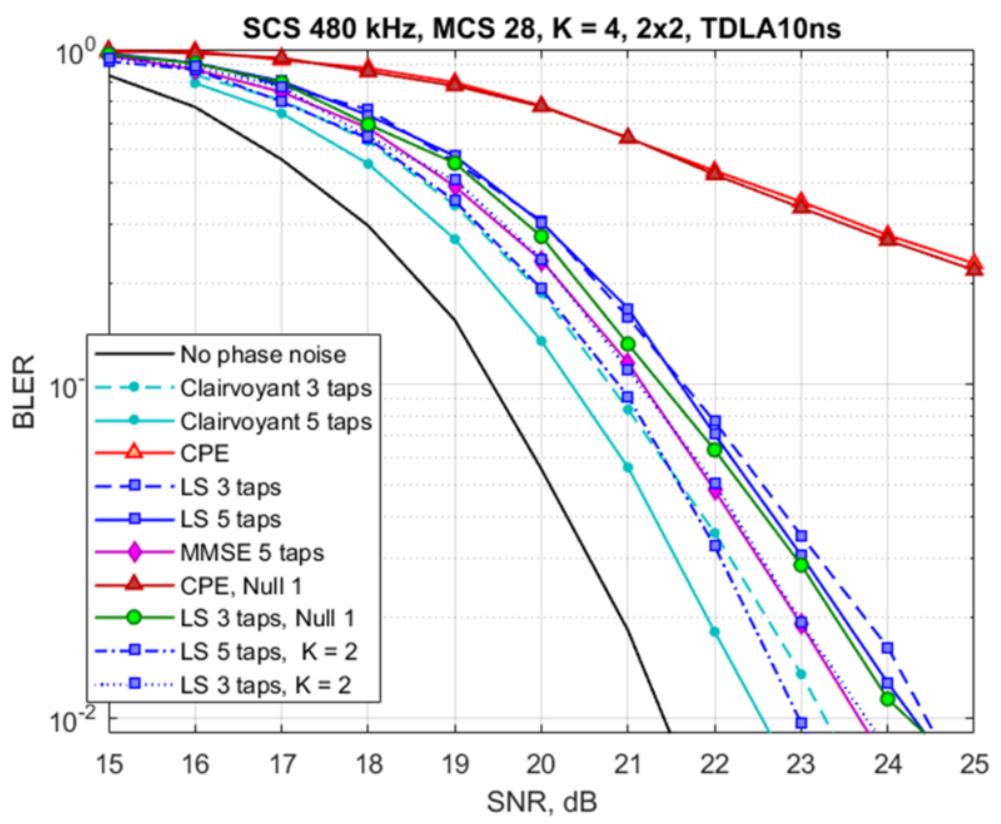

Figure 5 BLER vs. SNR performance comparison for SCS $480 \mathrm{kHz}$ 


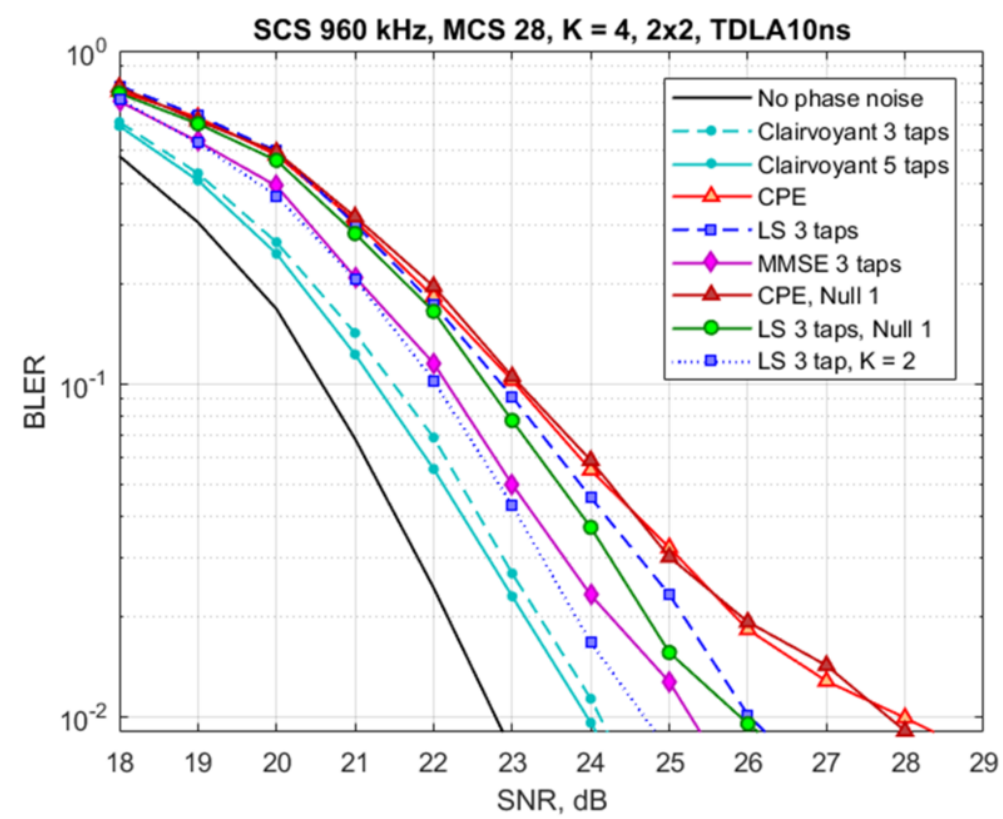

Figure 6 BLER vs. SNR performance comparison for SCS $960 \mathrm{kHz}$

Comparing the Clairvoyant mode with the LS algorithm for different SCS and Kparameters, it can be observed that there are two edge cases possible.

With relatively large number of PTRSs available (smaller SCS, smaller PTRS spacing $K=2$ ), the filter coefficient estimation accuracy is high, and the practical curves is very close to Clairvoyant ones for given number of taps, and the performance is limited with filter size. Compensation can be improved by increasing filter size; however, this will lead to a decrease in the coefficient estimation accuracy. Therefore, some balance is required between these parameters for achieving an optimal technical solution.

This leads to the opposite case when number of PTRSs is not enough for reliable estimation of the filter coefficients. This case is realized with larger SCS with fixed BW, larger PTRS spacing $(\mathrm{K}=4)$ and for the case of large number of filter taps to be estimated. In this case, we can even have the performance degradation in comparison with smaller filter size cases. So, for given number of available PTRSs there is some optimal filter size.

Near this optimal point, where filter estimation accuracy matters, we can improve the BLER performance by exploiting some advanced PTRS allocation schemes, such as nulling or estimation approaches (e.g., MMSE). It can be seen that MMSE can improve the performance in case of small number of PTRSs, but inefficient in case of higher density. At the same time, amount of large size matrices inversion which is required for MMSE calculation, makes it prohibitively complex for practical implementation. 
We can see that the difference between the best practical schemes and ideal Clairvoyant mode for given filter size may be as small as $0.5 \mathrm{~dB}$. At the same time, increasing the filter size provides diminishing gains with each increase, with simultaneous coefficients estimation accuracy dropped.

Proposed Nulling PTRS allocation scheme improves the filter taps estimation accuracy by partially avoiding the noise-induced ICI at pilots allocations and provides BLER improvement, about $0.2 \mathrm{~dB}$. It should be noted that BLER curves of the practical algorithms are pretty close to ones of the ideal Clairvoyant algorithm, so further improvement is not expected.

\section{Conclusion}

In this paper, we have investigated performance of various practical phase noise compensation algorithms operating on the baseline 5G NR PTRS structure and newly proposed PTRS with nulling allocation. It was shown that simple CPE compensation may not be enough for higher order MCS support in the presence of the phase noise, and ICI compensation algorithms are needed to make these modes feasible.

The performance of ICI compensation via Wiener filtering strongly depends on the filter size. It was shown that with dense PTRS placement, the LS and more computationally complex MMSE algorithm have similar performance, very close to the theoretical limit for given filter size.

In the practical case of limited number of available PTRSs, the key role in the compensation performance plays the filter coefficients estimation accuracy that can be increased either by using MMSE, or by exploiting proposed Nulling scheme that allows partial ICI avoidance. Although Nulling scheme provides minor gain about $0.2 \mathrm{~dB}$, it does not require any changes in the LS algorithm and provides a comparably small improvement in computational complexity, due to nulling part of the PTRSs used in calculations.

Generally, the phase noise compensation efficiency depends on the compensation filter size, and with the appropriate number of PTRSs, the increased filter size can be reliably evaluated. However, increasing filter size provides diminishing gains, due to quickly decaying of the phase noise PSD, whilst increasing the PTRS overhead gives stable spectral efficiency drop. Finding the combinations of the filter size and PTRS density, that maximizes system spectral efficiency may be the direction for further studies. Besides, as we demonstrated that the practical filtering ICI compensation techniques are very close in performance to the ideal Clairvoyant algorithm; other approaches like time-domain phase compensation should also be considered.

\section{Appendix A}

For the implementation of the MMSE algorithm, knowledge of the phase noise correlation matrix in frequency domain is required. By definition:

$$
\mathbf{R}_{J J}(p, q)=E\left\{J_{p} J_{q}^{*}\right\}=\frac{1}{N^{2}} \sum_{m=0}^{N-1} \sum_{n=0}^{N-1} E\left\{e^{j\left(\phi_{n}-\phi_{m}\right)}\right\} e^{\frac{-j 2 \pi}{N}(p n-q m)}
$$


The expression under the sum, the mathematical expectation of the random value exponent $E\left\{e^{j\left(\phi_{n}-\phi_{m}\right)}\right\}$ has the meaning of the characteristic function [13] at parameter $\mathrm{t}=1$ of the Gaussian process. Whole expression, in short is a 2D FFT from this characteristic function. So, we can find that:

$$
\left.E\left\{e^{j t\left(\phi_{n}-\phi_{m}\right)}\right\}\right|_{t=1}=\psi(1)=e^{-\sigma_{m n}^{2} / 2},
$$

where $\psi(t)=\exp \left\{\mu i t-\frac{\sigma^{2}}{2} t^{2}\right\}$ is Gaussian characteristic function [14].

The expression under the exponent $\sigma_{m n}^{2}$ is a dispersion of the difference between delayed samples of phase noise process that depends on autocorrelation function.

$$
\sigma_{m n}^{2}=\sigma_{m}^{2}+\sigma_{n}^{2}-2 \rho_{m n} \sigma_{m} \sigma_{n}=2 \sigma^{2}\left(1-\rho_{m n}\right)=2 \mathrm{~A}(0)-2 \mathrm{~A}(|m-n|)
$$

An autocorrelation function of the phase noise process is a Fourier transform from the PSD, which is a base of the phase noise model and generally should be known for given transmitter and receiver.

Figure A1 shows the structure of the investigated correlation matrix. It can be seen that only the center values can be taken into account while MMSE algorithm is used.

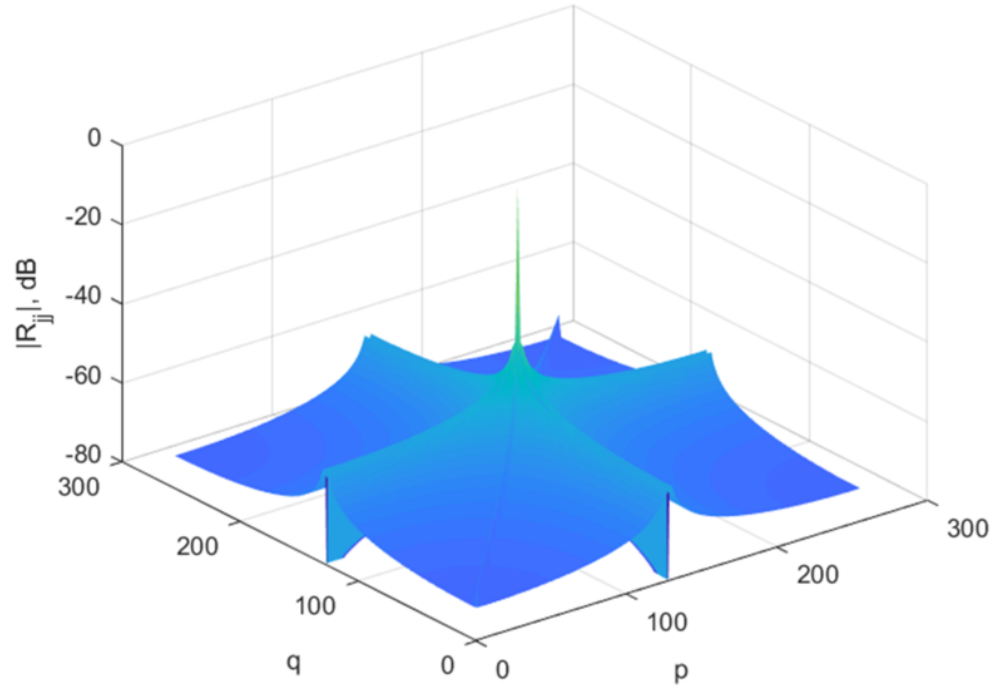

Figure A1 Phase noise frequency domain correlation matrix

\section{References}

[1] RP-202925, "Revised WID: on Extending current NR operation to $71 \mathrm{GHz} "$

[2] Maltsev, A., Maslennikov, R., Khoryaev, A. Influence of phase noise on OFDM data transmission systems. Radiophysics and Quantum Electronics, 2011, vol. 53, no. 8, p. 475-487.

[3] 3GPP TR 38.214 V15.10.0 (2020-06), "Physical layer procedures for data (Release 15)"

[4] Petrovic, D., Rave, W., and Fettweis, G., "Intercarrier interference due to phase noise in OFDMEstimation and suppression," in Proc. IEEE VTC Fall, Sep. 2004, pp. 2191-2195.

[5] Syrjälä, V., Levanen, T., Ihalainen T., and Valkama, M., "Pilot Allocation and Computationally Efficient Non-Iterative Estimation of Phase Noise in OFDM," IEEE Wireless Comm Letters, Apr. 2019. 
[6] Petrovic, D., Rave, W. and Fettweis, G. , "Effects of Phase Noise on OFDM Systems With and Without PLL: Characterization and Compensation,” IEEE Trans. Comm., Aug. 2007.

[7] T. Levanen, O. Tervo, K. Pajukoski, M. Renfors and M. Valkama, "Mobile Communications Beyond 52.6 GHz: Waveforms, Numerology, and Phase Noise Challenge," in IEEE Wireless Communications, vol. 28, no. 1, pp. 128-135, February 2021, doi: 10.1109/MWC.001.2000185.

[8] Y. Qi, M. Hunukumbure, H. Nam, H. Yoo and S. Amuru, "On the Phase Tracking Reference Signal (PTRS) Design for 5G New Radio (NR)," 2018 IEEE 88th Vehicular Technology Conference (VTC-Fall), 2018, pp. 1-5, doi: 10.1109/VTCFall.2018.8690852.

[9] 3GPP TS38.211V15.8.0(2019-12) "Physical channels and modulation (Release 15)"

[10] 3GPP TR 38.803 V14.2.0 (2017-09) "Study on new radio access technology: Radio Frequency (RF) and co-existence aspects"

[11] Lomayev, A.,Kravtsov, V., Genossar, M., Maltsev, A., Khoryaev, A., "Method for Phase Noise Impact Compensation in 60 GHz OFDM Receivers", Radioengineering, vol. 29, no. 1, April 2020

[12] R1-2005922 "On Phase Noise Compensation for OFDM"

[13] Proakis, John G., and Dimitris G. Manolakis. Digital Signal Processing. 3rd ed. Upper Saddle River, NJ: Prentice Hall, 1996.

[14] Kay, S. M. Fundamentals of Statistical Signal Processing. Vol. 1. Englewood Cliffs, NJ: PrenticeHall, 1998. ISBN: 0-13-345711-7

[15] 3GPP TR 38.901 V16.1.0 (2019-12), "Study on channel model for frequencies from 0.5 to $100 \mathrm{GHz}$ (Release 16)"

[16] 3GPP TR 38.212 V15.9.0 (2020-06), "Multiplexing and channel coding (Release 15)" 